\title{
Sudden Change in the Nuclear Charge Distribution of Very Light Gold Isotopes
}

\author{
K. Wallmeroth, G. Bollen, A. Dohn, ${ }^{(a)}$ P. Egelhof, J. Grüner, F. Lindenlauf, and U. Krönert \\ Institut für Physik, Unicersität Mainz, D-6500 Mainz, Federal Republic of Germany
}

J. Campos and A. Rodriguez Yunta

Universidad Complutense and Junta de Energía Nuclear, Madrid, Spain

M. J. G. Borge and A. Venugopalan ${ }^{(b)}$

ISOLDE Collaboration, CERN, CH-1211 Geneva, Switzerland

J. L. Wood

School of Physics, Georgia Institute of Technology, Atlanta, Georgia 30332

R. B. Moore

McGill Unicersity, Montreal, Canada H3A2B2

and

H.-J. Kluge

Institut für Physik, Universität Mainz, D-6500 Mainz, Federal Republic of Germany, and ISOLDE Collaboration, CERN, CH-1211 Geneva, Switzerland (Received 29 December 1986)

\begin{abstract}
On-line resonance-ionization mass spectrometry has been applied to determine the isotope shift and hyperfine structure of ${ }^{185-189} \mathrm{Au}$ and ${ }^{189} \mathrm{Au}^{m}$ in the $6 s^{2} S_{1 / 2} \rightarrow 6 p^{2} P_{1 / 2}(\lambda=268 \mathrm{~nm})$ transition. The Au atoms were obtained as daughters of mass-separated $\mathrm{Hg}$ isotopes produced at the ISOLDE facility at CERN ionized by a three-color, two-step resonant photoionization process, detected and mass selected by time of flight. A drastic change of the nuclear charge radius was observed between ${ }^{187} \mathrm{Au}$ and ${ }^{186} \mathrm{Au}$, which is interpreted as an onset of strong deformation of $\beta \approx 0.25$ in ${ }^{186} \mathrm{Au}$ and ${ }^{185} \mathrm{Au}$.
\end{abstract}

PACS numbers: $21.10 . \mathrm{Ft}, 21.10 . \mathrm{Ky}, 27.70 . \mathrm{tg}$

The nuclei near $Z=80$ and $N=104$ have attracted considerable attention following the discovery by Bonn et al. ' that there is a big change in the ground-state meansquare charge radius between ${ }^{187} \mathrm{Hg}$ and ${ }^{185} \mathrm{Hg}$, and its interpretation as an onset of strong deformation at $A \leq 185$. This result, obtained from a measurement of the optical isotope shift (IS), was completely unexpected since at that time the unanimous view was that nuclei near closed shells, such as the isotopes of $\mathrm{Hg}(Z=80)$, are only weakly deformed. This view and our knowledge of the region around ${ }^{185} \mathrm{Hg}$ have since changed dramatically.

It is becoming evident that coexisting shapes occur widely throughout the $\mathrm{Hg}$ region. This is manifested in the $\mathrm{Hg}$ isotopic chain by a large and systematic odd-even staggering in the root-mean-square charge radii of the very neutron-deficient $\mathrm{Hg}$ isotopes, ${ }^{2,3}$ a huge isomer shift ${ }^{4}$ in ${ }^{185} \mathrm{Hg}$, and strongly deformed bands coexisting at low excitation energy in the even-mass isotopes. ${ }^{5,6}$ In $\mathrm{Pt}$, a similar (but more complicated) situation has been deduced in the even-mass light isotopes ${ }^{7}$ and a large odd-even staggering has been found in the rotational spacings of the ground-state bands of the very neutrondeficient $\mathrm{Pt}$ isotopes. ${ }^{8}$ In the neighboring odd-proton nuclei ( $\mathrm{Au}$ and $\mathrm{Tl}$ isotopes) the shape coexistence is as- sociated with shell-model intruder states. ${ }^{5}$ In particular, the $\pi h_{9 / 2}$ intruder orbital becomes the ground-state configuration in ${ }^{185} \mathrm{Au}$ (see Desthuilliers et al. ${ }^{9}$ and

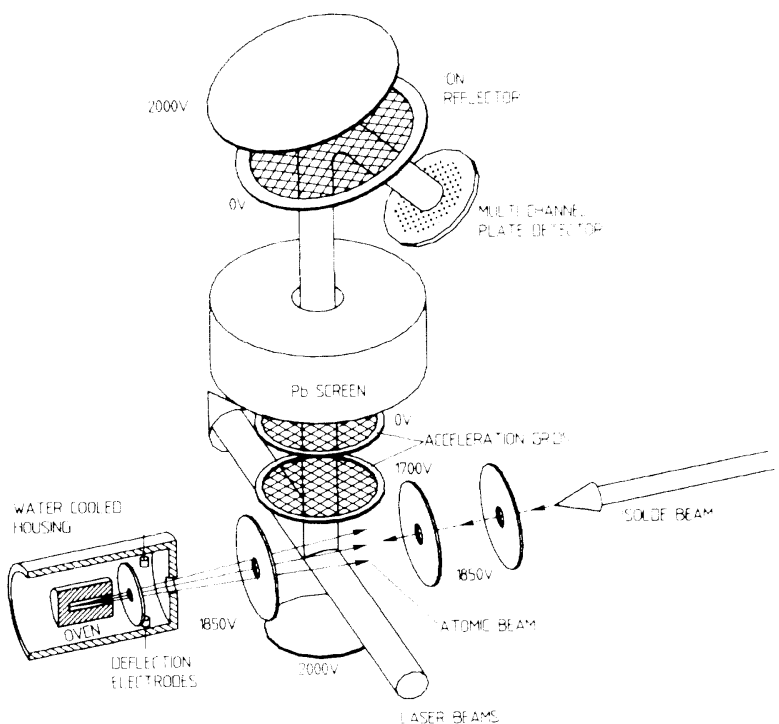

FIG. 1. Setup for on-line resonance ionization mass spectroscopy on short-lived Au isotopes. 
TABLE I. Optical transitions used for resonance ionization spectroscopy on short-lived Au isotopes, and data of the different lasers and laser beams $(\mathrm{SH}=$ second harmonic $)$.

\begin{tabular}{|c|c|c|c|c|c|c|c|}
\hline $\begin{array}{c}\text { Excita- } \\
\text { tion } \\
\text { step }\end{array}$ & $\begin{array}{l}\text { Optical } \\
\text { transition }\end{array}$ & $\begin{array}{c}\text { Wave- } \\
\text { length } \\
(\mathrm{nm})\end{array}$ & Light source & Dye & $\begin{array}{c}\text { Pulse } \\
\text { energy } \\
(\mu \mathrm{J})\end{array}$ & $\begin{array}{c}\text { Pulse } \\
\text { duration } \\
\text { (ns) }\end{array}$ & $\begin{array}{c}\text { Band- } \\
\text { width } \\
(\mathrm{GHz})\end{array}$ \\
\hline & $\cdots$ & 535 & Molectron DL16 $\mathrm{P}^{\mathrm{a}}$ & C540A & $10^{3}$ & 6 & $\leq 0.2$ \\
\hline No. 1 & $6 s^{2} S_{1 / 2} \rightarrow 6 p^{2} P_{1 / 2}$ & 268 & $\mathrm{SH}$ of $535 \mathrm{~nm}$ & $\cdots$ & 25 & 4 & $\leq 0.4$ \\
\hline No. 2 & $6 p^{2} P_{1 / 2} \rightarrow 6 d^{2} D_{3 / 2}$ & 407 & Lambda FL2001 & DPS & 150 & 6 & 6 \\
\hline No. 3 & $6 d^{2} D_{3 / 2} \rightarrow$ continuum & 532 & $\mathrm{SH}$ of $\mathrm{Nd}: \mathrm{YAG}$ & $\cdots$ & $4 \times 10^{4}$ & 15 & 15 \\
\hline
\end{tabular}

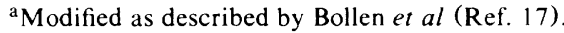

Ekström et al. ${ }^{10}$ ) whereas in the odd-mass $\mathrm{Tl}$ isotopes the $\pi h_{9 / 2}$ intruder state remains an excited state. For the latter case, isomerism results from this $\frac{9}{2}$ state, which has been studied recently by laser spectroscopy. ${ }^{11}$ It thus became interesting to extend the IS studies to neutron-deficient gold isotopes. In an earlier experiment ${ }^{12}$ the IS's of ${ }^{197-190} \mathrm{Au}$ were determined by use of semi-on-line laser-induced fluorescence spectroscopy.

The present experiment was designed to investigate still shorter-lived $\mathrm{Au}$ isotopes and to determine their IS's, which represent the most straightforward and model-independent signature for shape transitions or coexistence. The $\mathrm{Au}$ isotopes were obtained at the ISOLDE mass separator at CERN, as daughters of $\mathrm{Hg}$ isotopes which could be produced with yields up to $10^{10}$ atoms per second per mass number by a $\mathrm{Pb}(p, 3 p x n) \mathrm{Hg}$ spallation reaction. ${ }^{13}$ The technique of resonance ionization spectroscopy ${ }^{14,15}$ was used and combined with timeof-flight mass spectroscopy. Alkhazov et al. ${ }^{16}$ performed the first on-line resonance-ionization-spectroscopy experiment on short-lived isotopes. The present Letter reports the first on-line application of resonance-ionization-mass spectroscopy.

Figure 1 shows the experimental setup. The ISOLDE beam was focused into an atomic beam oven. After a suitable time had elapsed during which the $\mathrm{Hg}$ nuclei decayed to the daughter isotope, a thermal atomic beam was formed by evaporating the radioactive sample. The $\mathrm{Au}$ atoms were excited and photoionized via a three- color, two-step resonant process by the light from two tunable dye lasers pumped by a Nd-doped: yttrium aluminum garnet (YAG) laser and the frequencydoubled output of the same $\mathrm{Nd}$ :YAG laser (repetition rate $=10 \mathrm{~Hz}$ ). The optical transitions used for this experiment are shown in Table I. In order to increase the signal-to-background ratio, the photoions were detected and mass selected by a time-of-flight spectrometer. ${ }^{18}$ An ion reflector was installed to allow an efficient shielding of the multichannel plate detector against nuclear radiation from the oven/acceleration region. A background rate as low as 1 event per 1000 laser shots was obtained with a time-of-flight mass resolution of 250 and a gate of 70 -ns width on the mass of the isotope under investigation. The overall detection efficiency of our resonanceionization-mass-spectrometry technique for $\mathrm{Au}$ (defined as the ratio of $\mathrm{Au}$ ions detected by the multichannel plate to the number of atoms collected in the oven) was about $10^{-8}$, mainly limited by the low repetition rate of the laser system.

Data on the hyperfine structure (hfs) and IS in the $D_{1}$ line of ${ }^{185-189} \mathrm{Au}$ and ${ }^{189} \mathrm{Au}^{m}$ were obtained. The IS of ${ }^{190} \mathrm{Au}$ was remeasured and agrees within error bars with the value cited in the earlier study. ${ }^{12}$ The magnetic moments deduced are compiled in Table II. The agreement with earlier measurements ${ }^{10,20}$ is good, except for ${ }^{187} \mathrm{Au}$. The reason for this discrepancy is not understood.

Table III gives the results obtained from the determination of the IS in the $D_{1}$ line. The third column

TABLE II. Spins, ground-state hyperfine constants, and magnetic moments of short-lived $\mathrm{Au}$ isotopes.

\begin{tabular}{cccccc}
\hline \hline & & \multicolumn{2}{c}{ This work $^{\mathrm{a}}$} & \multicolumn{2}{c}{ Literature } \\
$A$ & $I$ & $A\left(6 s^{2} S_{1 / 2}\right)(\mathrm{MHz})$ & $\mu_{I} / \mu_{N}$ & $\mu_{I} / \mu_{N}$ & Reference \\
\hline 189 & $\frac{1}{2}$ & $+28632(128)$ & $+0.49(3)$ & $\ldots$ & $\ldots$ \\
$189 m$ & $\frac{11}{2}$ & $+32625(42)$ & $+6.19(3)$ & $\pm 6.19(12)$ & 19 \\
188 & 1 & $-1940(127)$ & $-0.07(3)$ & $\pm 0.069(24)$ & 18 \\
187 & $\frac{1}{2}$ & $+31032(168)$ & $+0.54(3)$ & $+0.72(7)$ & 18 \\
186 & 3 & $-12207(48)$ & $-1.26(3)$ & $\pm 1.07(13)$ & 19 \\
185 & $\frac{5}{2}$ & $+25176(56)$ & $+2.17(3)$ & $\pm 2.22(14)$ & 19 \\
\hline \hline
\end{tabular}

${ }^{a}$ Calculated from the magnetic HFS splitting in the $D_{1}$ line with the relation (Ref. 10) $\mu_{I}=(A I / 29005) \mu_{N}$. The experimental error accounts for the uncertainty in the HFS anomaly. 
TABLE III. Isotope shift and deduced data of the charge radii and deformations as obtained in the $D_{1}$ line of the short-lived $\mathrm{Au}$ isotopes. ${ }^{197} \mathrm{Au}$ is taken as the reference isotope. The errors given are purely experimental and do not account for the (common scaling) uncertainty of $F_{268}$.

\begin{tabular}{|c|c|c|c|c|c|c|}
\hline$A$ & $\begin{array}{c}T_{1 / 2} \\
(\mathrm{~min})\end{array}$ & $\begin{array}{l}\delta l^{1} \begin{array}{l}197, A \\
\text { expt }\end{array} \\
(\mathrm{GHz})\end{array}$ & $\begin{array}{l}\lambda^{197, A} \\
\left(\mathrm{fm}^{2}\right)\end{array}$ & $\begin{array}{c}\delta\left\langle r^{2}\right\rangle{ }^{197, A}, A \\
\left(\mathrm{fm}^{2}\right)\end{array}$ & $\delta\left\langle\beta^{2}\right\rangle^{197, A}$ & $\left\langle\beta^{2}\right\rangle^{1 / 2}$ \\
\hline 190 & 42.8 & $11.39(16)$ & $-0.267(6)$ & $-0.285(6)$ & 0.007 & 0.14 \\
\hline 189 & 28.3 & $12.35(18)$ & $-0.290(7)$ & $-0.309(7)$ & 0.010 & 0.15 \\
\hline $189 m$ & 4.6 & $11.36(22)$ & $-0.267(8)$ & $-0.283(9)$ & 0.012 & 0.15 \\
\hline 188 & 8.8 & $13.91(23)$ & $-0.327(8)$ & $-0.347(9)$ & 0.011 & 0.15 \\
\hline 187 & 8.0 & $15.40(23)$ & $-0.362(9)$ & $-0.385(9)$ & 0.012 & 0.16 \\
\hline 186 & 10.7 & $0.84(17)$ & $-0.025(8)$ & $-0.014(8)$ & 0.048 & 0.25 \\
\hline 185 & 4.2 & $3.26(18)$ & $-0.081(8)$ & $-0.075(9)$ & 0.048 & 0.24 \\
\hline
\end{tabular}

shows the experimental IS's relative to ${ }^{197} \mathrm{Au}$. After correction for the normal mass shift and taking into account an estimate ${ }^{21}$ for the specific mass shift, the remaining field shift $\delta v_{\mathrm{FS}}^{A, A^{\prime}}$ were related by $\delta v_{\mathrm{FS}, 268}^{A, A^{\prime}}$ $=F_{268} \lambda^{A, A^{\prime}}$ to the nuclear parameter

$$
\lambda^{A, A^{\prime}}=\sum\left(C_{n} / C_{1}\right) \delta\left\langle r^{2 n\rangle} A, A^{\prime},\right.
$$

where $F_{268}$ is the electronic factor for the $D_{1}$ transition. The parameters $F_{268} \equiv C_{1}=-43.07 \mathrm{GHz} / \mathrm{fm}^{2}$ and $C_{n} / C_{1}$ were calculated with multiconfigurational DiracFock theory. ${ }^{22}$ The resulting model-independent $\lambda$ parameters are shown in the fourth column of Table III. Column five of Table III and Fig. 2 show the $\delta\left\langle r^{2}\right\rangle$ values, calculated as in the case of $\mathrm{Hg}$ (Ref. 3) and $\mathrm{Eu}$ (see Ahmad et al. ${ }^{23}$ ). The slope of the mean square charge radii is monotonic down to ${ }^{187} \mathrm{Au}$ and is similar to the one of the $\mathrm{Hg}$ isotopes. Between ${ }^{187} \mathrm{Au}$ and ${ }^{186} \mathrm{Au}$, a drastic change occurs, similar to the $\mathrm{Hg}$ isotopes but two neutron numbers earlier. There is a much less pronounced odd-even staggering for the Au isotopes than for the $\mathrm{Hg}$ isotopes. As in the $\mathrm{Hg}$ isotopes, the break in the IS can be related to a change in nuclear quadrupole de-

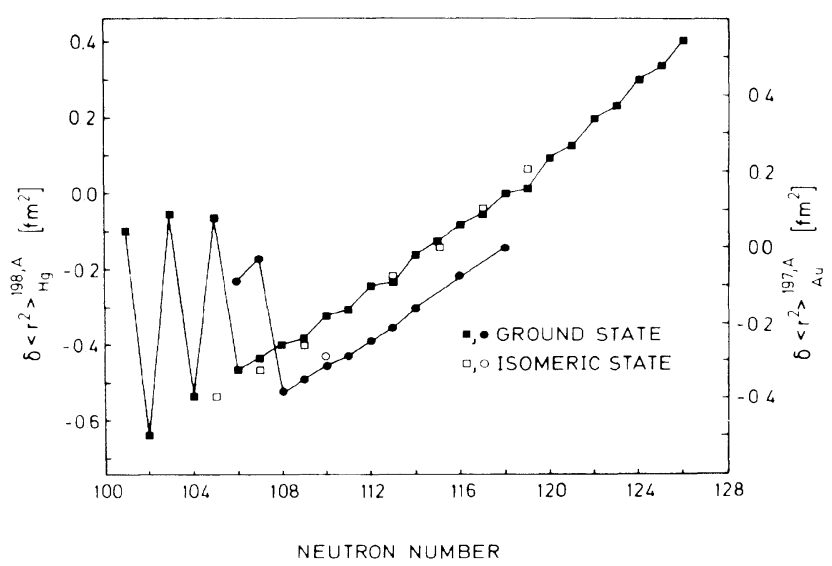

FIG. 2. Changes of the mean square charge radii of $\mathrm{Au}$ (circles: this work and Ref. 21) and of $\mathrm{Hg}$ (squares: Ref. 11) isotopes. formation $\beta$ by

$$
\delta\left\langle r^{2}\right\rangle=\delta\left\langle r^{2}\right\rangle_{\mathrm{sph}}+\delta\left\langle r^{2}\right\rangle_{\mathrm{def}}
$$

and

$$
\delta\left\langle r^{2}\right\rangle_{\mathrm{def}}=(5 / 4 \pi)\left\langle r^{2}\right\rangle_{\mathrm{sph}} \delta\left\langle\beta^{2}\right\rangle
$$

The values $\delta\left\langle r^{2}\right\rangle_{\text {sph }}$ are taken from the droplet model, ${ }^{24}$ which is known to describe well the spherical part of the change of the nuclear charge radius in the mass region under consideration. The resulting changes in the rms deformation parameters are compiled in Table III. Absolute deformation parameters can be evaluated if $\beta$ is known for at least one isotope. As in the previous publication, ${ }^{12}$ we take $\left|\beta\left({ }^{197} \mathrm{Au}\right)\right|=0.11$, which leads to the values given in the last column of Table III.

The values of $\left\langle\beta^{2}\right\rangle^{1 / 2}$ in Table III clearly imply the sudden onset of deformation in going from ${ }^{187} \mathrm{Au}$ to ${ }^{186} \mathrm{Au}$, with its persistence of ${ }^{185} \mathrm{Au}$. The change between ${ }^{187} \mathrm{Au}$ and ${ }^{186} \mathrm{Au}$ is also reflected in $B(E 2)$ values for transitions feeding the ground states (see Berg and co-workers ${ }^{25,26}$ and Abreu et al. ${ }^{27}$ ). The deduced deformation of ${ }^{185} \mathrm{Au}$ (see Table III) confirms the recent prediction $^{20} \quad(\beta=0.25)$. The observed shape change between ${ }^{187} \mathrm{Au}$ and ${ }^{185} \mathrm{Au}$ can also be understood simply in the intruder state picture ${ }^{5}$ as the difference between the core-particle couplings $\pi^{-1} \otimes{ }^{188} \mathrm{Hg}$ and $\pi^{+1} \otimes{ }^{184} \mathrm{Pt}$, respectively. In the heavy gold isotopes the ground-state configuration is $\pi d_{3 / 2}^{-1}$; however, between ${ }^{187} \mathrm{Au}$ and ${ }^{185} \mathrm{Au}$ the $\pi h_{9 / 2}^{+1}$ configuration intrudes ${ }^{5}$ to become the ground state. In ${ }^{187} \mathrm{Au}$ the $d_{3 / 2}$ proton hole couples to a ${ }^{188} \mathrm{Hg}$ core for which a small deformation $\beta=(-) 0.15$ has been determined, ${ }^{3}$ in ${ }^{185} \mathrm{Au}$ the $\pi h_{9 / 2}^{+1}$ proton particle couples to a ${ }^{184} \mathrm{Pt}$ core for which $\beta=(+) 0.24$ can be deduced from the $B\left(E 2 ; 2^{+} \rightarrow 0^{+}\right)$value. ${ }^{28}$ The absence of a strong odd-even staggering between ${ }^{185} \mathrm{Au} /{ }^{186} \mathrm{Au}$ as it has been observed in the chain of the $\mathrm{Hg}$ isotopes starting with ${ }^{185} \mathrm{Hg}$ (see Fig. 2) can be qualitatively understood as the result of the $\pi h_{9 / 2}^{+1}$ intruder configuration being the ground-state proton configuration in both ${ }^{185} \mathrm{Au}$ and ${ }^{186} \mathrm{Au}$. This confirms the conclusion of Porquet et al. ${ }^{29}$ based on a study of excited states in ${ }^{186} \mathrm{Au}$ and on a comparison with the excited-states systematics 
of the heavier odd-odd gold isotopes.

This work has been funded by the German Federal Minister for Research and Technology (Bundesminister für Forschung und Technologie) under Contract No. MZ-458-1. One of us (J.L.W.) was supported in part by U.S. Department of Energy Contract No. DE-AS0580ER 10599. This Letter comprises part of one author's (K.W.) thesis.

(a) Present address: W. C. Heraeus GmbH, D-6450 Hanau, Federal Republic of Germany.

(b) On leave from the Bhabha Atomic Research Centre, Bombay 400085 , India.

${ }^{1}$ J. Bonn et al., Phys. Lett. 38B, 308 (1972), and Z. Phys. A 276, 203 (1976).

${ }^{2}$ T. Kühl et al., Phys. Rev. Lett. 39, 180 (1977).

${ }^{3}$ G. Ulm et al., Z. Phys. A 325, 247 (1986).

${ }^{4}$ P. Dabkiewicz et al., Phys. Lett. 82 B, 199 (1979).

${ }^{5} \mathrm{~K}$. Heyde et al., Phys. Rep. 102, 291 (1983).

${ }^{6}$ J. H. Hamilton et al., Rep. Prog. Phys. 48, 631 (1985).

${ }^{7}$ G. D. Dracoulis et al., J. Phys. G 12, L97 (1986).

${ }^{8}$ E. Hagberg et al., Phys. Lett. 78 B, 44 (1978).

${ }^{9}$ M. G. Desthuilliers et al., Nucl. Phys. A313, 221 (1979).

${ }^{10}$ C. Ekström et al., Nucl. Phys. A348, 25 (1980).
11 J. A. Bounds et al., Phys. Rev. Lett. 55, 2269 (1985).

12 J. Streib et al., Z. Phys. A 321, 537 (1985).

${ }^{13}$ ISOLDE Users' Guide, edited by H.-J. Kluge, CERN Report No. 86-05, 1986 (unpublished).

${ }^{14} \mathrm{~V}$. S. Letokhov, Nonlinear Laser Chemistry, Springer Series Chemistry Physics Vol. 22 (Springer-Verlag, Berlin, 1984).

${ }^{15}$ G. S. Hurst et al., Rev. Mod. Phys. 51, 767 (1979).

${ }^{16} \mathrm{G}$. D. Alkhazov et al., JETP Lett. 37, 274 (1983).

${ }^{17} \mathrm{G}$. Bollen et al., to be published.

${ }^{18}$ U. Krönert et al., Appl. Phys. B 38, 65 (1985).

${ }^{19} \mathrm{~L}$. L. Riedinger et al., in Proceeding of the Symposium on Recent Advances in the Study of Nuclei off the Line of Stability, Chicago, Illinois, 1985, edited by R. A. Meyer and D. S. Brenner (to be published).

${ }^{20}$ E. van Walle et al., Hyperfine Interact. 22, 507 (1985).

${ }^{21} \mathrm{~K}$. Heilig and A. Steudel, At. Data Nucl. Data Tables 14, 613 (1974).

${ }^{22}$ A. Rosén et al., Z. Phys. A 316, 157 (1984).

${ }^{23}$ S. A. Ahmad et al., Z. Phys A 321, 35 (1985).

${ }^{24}$ W. D. Myers and K.-H. Schmidt, Nucl. Phys. A 410, 61 (1983).

${ }^{25}$ V. Berg et al., Nucl. Phys. A410, 445 (1983).

${ }^{26}$ V. Berg et al., Nucl. Phys. A453, 93 (1986).

${ }^{27}$ M. C. Abreu et al., Nucl. Phys. A437, 324 (1985).

${ }^{28}$ U. Garg et al., Phys. Lett. 180B, 319 (1986).

${ }^{29}$ M. G. Porquet et al., Nucl. Phys. A411, 65 (1983). 